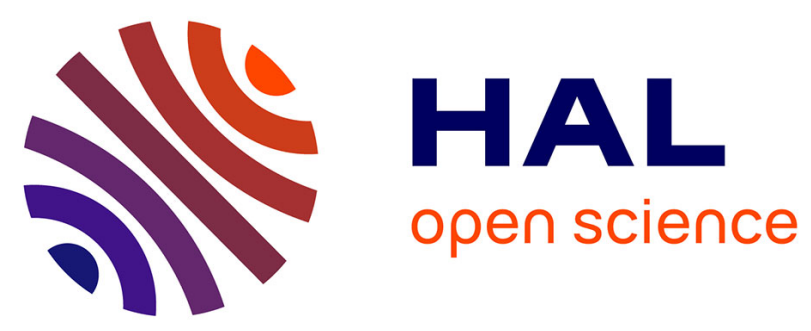

\title{
Nobles, aristocrates, châtelains : du pareil au même. Mobilisations contre une figure de l'ennemi et politisation des campagnes cantaliennes lors des troubles de mars 1792.
}

Sébastien Pivoteau

\section{To cite this version:}

Sébastien Pivoteau. Nobles, aristocrates, châtelains: du pareil au même. Mobilisations contre une figure de l'ennemi et politisation des campagnes cantaliennes lors des troubles de mars 1792.. Colloque international sur "Les Noblesses dans l'Europe de la Révolution (années 1770-1800)", Sep 2008, Vizille, France. p. 547-562. hal-00965223

HAL Id: hal-00965223

https://hal.uca.fr/hal-00965223

Submitted on 25 Mar 2014

HAL is a multi-disciplinary open access archive for the deposit and dissemination of scientific research documents, whether they are published or not. The documents may come from teaching and research institutions in France or abroad, or from public or private research centers.
L'archive ouverte pluridisciplinaire HAL, est destinée au dépôt et à la diffusion de documents scientifiques de niveau recherche, publiés ou non, émanant des établissements d'enseignement et de recherche français ou étrangers, des laboratoires publics ou privés. 


\section{Nobles, aristocrates, châtelains : du pareil au même. Mobilisations contre une figure de l'ennemi et politisation des campagnes cantaliennes lors des troubles de mars $\mathbf{1 7 9 2}^{1}$.}

Stigmatisée dans la presse et la littérature, pourfendue sur les scènes de théâtre, la figure du noble aristocrate est encore combattue, l'arme à la main, à l'occasion de certains troubles ruraux de la période révolutionnaire. La «célèbre insurrection du Cantal (HauteAuvergne) $»^{2}$ en apporte une preuve tangible : au cours de la deuxième quinzaine de mars 1792, une quarantaine de châteaux sont attaqués, principalement dans le district d'Aurillac ${ }^{3}$.

Des archives judiciaires récemment inventoriées ${ }^{4}$ autorisent une relecture des mécanismes de politisation alors à l'œuvre. En comparaison avec la documentation produite sur le moment par les autorités locales ${ }^{5}$, les procédures judiciaires ainsi exhumées pâtissent à l'évidence de leur éloignement des faits : l'information diligentée par les juges de paix du district d'Aurillac ne s'ouvre pas avant la dernière semaine d'avril, époque de l'arrivée des premières troupes régulières, et les jugements prononcés par les tribunaux de district s'échelonnent de mai à juillet. Mais, en dépit d'inévitables aspects contradictoires, les dépositions consignées par les greffiers livrent un tableau pittoresque des événements, qui éclaire d'un jour nouveau les rapports des ruraux à la politique.

Bastions nobiliaires conservant le souvenir du système seigneurial, demeures opulentes faisant injure aux privations quotidiennes et, il n'est plus permis d'en douter, repaires d'une Contre-Révolution menaçante, les châteaux cristallisent une exaspération dont les manifestations concrètes et symboliques trahissent une vive sensibilité à la conjoncture nationale. L'animosité générale contre la figure de l'ennemi aristocrate n'entraîne cependant

\footnotetext{
${ }^{1}$ La présente contribution reprend les grandes lignes de la deuxième partie d'un mémoire de Master 2 soutenu en 2008 à l'Université Blaise Pascal (Clermont-Ferrand II). Qu'il nous soit permis de remercier ici Philippe Bourdin, qui a bien voulu diriger ce travail et lui accorder une place à Vizille.

2 Anatoli ADO, Paysans en Révolution. Terre, pouvoir et jacquerie. 1789-1794, Paris, Société des Études Robespierristes, 1996 [traduction d'après la seconde édition russe (1987) d'une thèse publiée à Moscou en 1971], p. 264.

${ }^{3}$ Voir les cartes insérées en fin de contribution.

${ }^{4}$ Nicolas Laparra, Supplément à la série L. Archives judiciaires, Aurillac, Archives départementales du Cantal (A.D.C.), 2004.

${ }^{5}$ Les études consacrées au soulèvement cantalien de mars 1792 ont puisé l'essentiel de leur matériau dans les procèsverbaux de municipalités (conservés pour la plupart aux Archives nationales, sous la cote $\mathrm{F}^{7}$ 3662) et dans les délibérations des administrateurs du département et des districts (A.D.C., Série L, Fonds du département et des districts d'Aurillac et de Saint-Flour).
} 
nulle « unanimité communale $»^{6}:$ les sources judiciaires renvoient l'image de communautés roturières profondément divisées, au sein desquelles s'affirment, entre les discours, les menaces et les cris, deux visions concurrentes de la justice sociale, autrement dit deux lignes politiques.

Avant d'examiner ces deux versants de la politisation des campagnes cantaliennes, un rapide parcours historiographique permettra de préciser la trame événementielle des attaques de châteaux de 1792 .

\section{De la révolte primitive au mouvement politique : des interprétations divergentes sur le statut de la violence.}

\section{Des « brigands » manipulés par un « homme de sang ».}

L'histoire des troubles de mars 1792 a d'abord été écrite par des épigones d'Hippolyte Taine, qui dénient toute rationalité à des violences commises par des foules fanatisées. L'opuscule publié à la fin du XIX ${ }^{\mathrm{e}}$ siècle par le vicomte B. de Miramon-Fargues offre une formulation caractéristique de ce courant historiographique ${ }^{7}$.

Dans cette optique, l'insurrection cantalienne résulte d'un sinistre complot machiné par «quelques agitateurs»sans scrupules, sur lesquels «s'exerçait l'influence d'une seule commune et d'un seul homme, qui mirent à profit les germes de discordes éclos dans le pays, et sur lesquels doit être rejetée toute la responsabilité des événements de $1792 »^{8}$. Se trouve ainsi sur la sellette Jean-Baptiste Milhaud, commandant de la garde nationale d'Arpajon et principal maître d'œuvre, au printemps et à l'été 1791, d'une Société des Hommes de la Nature ou Société patriotique et agricole des francs-tenanciers des campagnes et d'une fédération des gardes nationaux de la plupart des communes du canton d'Aurillac. Après son échec aux législatives de septembre 1791, Milhaud aurait prémédité une conspiration, qui devait éclater le 11 mars $1792^{9}$.

\footnotetext{
6 Pour reprendre le titre d'un développement relatif aux «troubles révolutionnaires », dans Yves-Marie BERCE, Croquants et nu-pieds. Les soulèvements paysans en France du XVI au XIX siècle, Paris, Gallimard, 1991 [1 ère édition : 1974], p. 175.

${ }^{7}$ Bernard de Miramon-Fargues, Épisodes de la Révolution dans le Cantal. La Jacquerie de 1792, Aurillac, Imprimerie H. Gentet, 1891. La même tonalité dénonciatrice imprègne l'Histoire de la Révolution en Auvergne de l'abbé Jean-Baptiste SERRES (dix volumes publiés entre 1895 et 1899), et se rencontre encore dans plusieurs Scènes de la Révolution dans le Cantal rapportées par le chanoine Édouard JOUBERT (Aurillac, Imprimerie moderne, 1972 ; voir en particulier les chapitres I, IV, XXI et XXV).

${ }^{8}$ Bernard de Miramon-Fargues, Épisodes..., op. cit., p. 7.

${ }^{9}$ Ibid., p. $18-23$.
} 
Ce jour-là, deux à trois mille gardes nationaux du canton sont rassemblés à Aurillac pour le recrutement de volontaires. Dans la nuit, un incident se produit sous les fenêtres de Colinet de Niocel, lieutenant criminel au présidial pendant quarante ans. Quelques volontaires chantant le «ça ira » reçoivent divers projectiles et essuient même un coup de pistolet. Le lendemain matin, une plainte officielle est déposée auprès de la municipalité, qui fait aussitôt arrêter Colinet. Mais la nouvelle rapidement répandue dans les environs, grossie de rumeurs de subversion aristocratique à Aurillac, mobilise les gardes nationales rurales. Après s'être réunies à Arpajon, celles-ci marchent au secours des patriotes aurillacois, Milhaud en tête, et quelques heures plus tard, Colinet est tiré de prison et décapité.

S'ensuivent trois semaines d'agitation connues sous le nom d'«Illuminations arpajonnaises $»^{10}$, dont la « guerre aux châteaux » « fut l'aspect (...) qui produisit la plus forte impression, celui qui allait laisser la plus longue trace dans les mémoires $»^{11}$. A en croire $\mathrm{B}$. de Miramon-Fargues, la quasi-totalité des exactions perpétrées alors est imputable aux «forces de la Jacquerie $»^{12}$ commandées par Milhaud, «homme de sang $»^{13}$ dont les discours enflammés, loin de nourrir une quelconque conscience politique, ont fourni avant tout un prétexte commode permettant de «dissimuler sa cupidité derrière des apparences un peu moins inavouables $»^{14}$ :

Il est intéressant de remarquer que les choses se passaient presque toujours correctement, lorsque les municipalités étaient abandonnées à elles-mêmes, et que le désordre était généralement provoqué par l'invasion des étrangers ${ }^{15}$.

\section{Des acteurs rationnels sensibles à la conjoncture nationale.}

Contre cette vision de sauvages envoûtés par un arriviste, plusieurs historiens ${ }^{16}$, dans le droit fil des travaux de Georges Lefebvre, ont mis en évidence l'autonomie relative de ruraux dotés d'une conscience collective.

\footnotetext{
${ }^{10}$ D'après Bernard de Miramon-FARGUeS, « Milhaud et ses amis » désignaient ainsi les incendies de châteaux qu'ils provoquaient (Épisodes..., op. cit., p. 36).

${ }^{11}$ Jonathan R. DALBY, Les Paysans cantaliens et la Révolution française (1789-1794), Clermont-Ferrand, Institut d'Études du Massif Central, 1989 [thèse de l’Université de Manchester soutenue en 1981], p. 77.

12 Bernard de Miramon-Fargues, Épisodes..., op. cit., p. 38.

${ }^{13}$ Ibid., p. 16.

${ }^{14}$ Ibid., p. 45.

15 Ibid., p. 39.

${ }^{16}$ Deux d'entre eux ont centré leurs analyses sur le département du Cantal : Michel LEYMARIE, «Les troubles ruraux en Haute-Auvergne et dans le Cantal de 1789 à 1795 » [article paru à l'origine dans Revue de la Haute-Auvergne, 19551962], dans M. LEYMARIE, La Haute-Auvergne de l'Ancien Régime à la Révolution, t. II, Aurillac, Société «La HauteAuvergne », 1994, p. 83-113 ; Jonathan R. DALBY, Les Paysans cantaliens..., op. cit. (voir en particulier le chapitre III, consacré aux « jacqueries de 1792 ». Deux autres historiens ont inscrit leur examen de la situation cantalienne dans un contexte régional élargi ou national : Anatoli ADO, Paysans en Révolution..., op. cit., p. 261-275 et 292-306; Hubert
} 
Revenant sur le déroulement même de l'insurrection, ils montrent que celle-ci n'a pas eu un centre unique, mais au moins trois foyers. Dans les jours qui suivent le meurtre de Colinet de Niocel, des «attroupements spontanés ${ }^{17}$ se forment dans les communes rurales proches d'Aurillac, avant que Milhaud n'assemble sa fédération de gardes nationales, le dimanche 18 mars, pour mener une opération punitive dans le canton de Montsalvy, bastion de l’Église réfractaire. Un quartier général est établi à mi-chemin entre Arpajon et Montsalvy, d'où sont envoyées des unités de gardes dans les châteaux du voisinage. Dès le 21 mars, l'opération est achevée, et les troupes de Milhaud peuvent regagner leurs foyers dans le canton d'Aurillac. A s'en tenir à la trame des faits, la responsabilité des «Arpajonnais » ne saurait donc être engagée en dehors de la partie orientale du district d'Aurillac. Plus à l'ouest, dans le canton de Maurs, «le maire de Boisset, Hugues Lac, comprenant tout l'avantage politique à retirer d'un soutien à la cause paysanne, dirigea lui-même ses propres opérations $»^{18}$, jusque dans des communes voisines. Enfin, « Le district de Saint-Flour connut aussi des semaines agitées, et pourtant Milhaud et les Arpajonnais n'y faisaient point la loi $! »^{19}$

Il serait néanmoins réducteur d'apprécier l'influence du commandant d'Arpajon à la seule aune de son implication au moment du soulèvement de mars 1792. Ses efforts de l'année précédente pour mettre en place un réseau de militants dans les campagnes au sud d'Aurillac ne sont certainement pas étrangers à la diffusion, sinon à l'explosion des troubles. Mais, sur ce terrain de l'acculturation politique, comme sur le théâtre des opérations militaires, Milhaud n'est pas seul. Parmi le «groupe de jacobins-démocrates (...) lié, directement ou indirectement, à l'insurrection dans le Cantal $»^{20}$, peut être cité Pagès de Vixouze, dont le journal Le Cantaliste, paru entre décembre 1790 et décembre 1791, régale ses lecteurs de diatribes hautes en couleurs contre les nobles et les prêtres réfractaires ${ }^{21}$.

Celles-ci resteraient pourtant lettre morte si elles ne prenaient appui sur un faisceau de mécontentements, alimentés par des vexations et des insatisfactions locales, mais aussi par les insuffisances de la législation agraire et les menaces de guerre. La révolte du Cantal s'inscrit d'ailleurs dans un vaste mouvement de protestation rurale qui, entre février et mai 1792,

DeLPONT, La Victoire des croquants. Les révoltes paysannes du Grand Sud-Ouest pendant la Révolution (1789-1799), [Nérac], Amis du Vieux Nérac, 2002, p. 229-264 et 279-332.

17 Jonathan R. DALBY, Les Paysans cantaliens..., op. cit., p. 68.

18 Ibid., p. 68.

${ }^{19}$ Michel LeYMARIE, « Les troubles ruraux... », art. cit., p. 104.

20 Anatoli ADO, Paysans en Révolution..., op. cit., p. 298.

21 Voir l'introduction des présents actes, ainsi que Philippe BOURDIN, entrée «Le Cantaliste », dans Gilles FEYEL (dir.), Dictionnaire de la presse française pendant la Révolution, 1789-1799. La presse départementale, Ferney-Voltaire, Centre international d'étude du XVIII e siècle (volume à paraître). 
submerge de nombreux départements du Centre et du Midi, et dans lequel A. Ado a décelé une « cinquième jacquerie après celle de $1789 »^{22}$.

\section{Une imprégnation politique inégale?}

L'historien russe s'empresse d'ajouter que celle-ci «s'épanouissait de façon originale en un mouvement patriotique et révolutionnaire $»^{23}$ et que 《la participation directe et massive des paysans à la lutte sociale du côté de la Révolution atteignit [alors] son point culminant $»^{24}$.

Si l'on en croit J. R. Dalby, la «politisation » du soulèvement cantalien de mars 1792 demeure toutefois sujette à caution, dans la mesure où le jeu des antagonismes personnels et familiaux, comme la pratique répandue des contributions forcées, révèlent d'autres lignes de fractures que celle séparant «patriotes » et «aristocrates » :

(...) même si les "aristocrates" (qu'ils aient été de l'espèce politique, ecclésiastique ou bien noble) furent les premiers visés par l'insurrection, il ne faut pas vouloir tout expliquer par l'opposition entre "patriotes » et «aristocrates ». La masse des paysans et des gardes nationaux soulevés ne pouvait être animée par une motivation politique un peu complexe et, pour beaucoup d'entre eux, la jacquerie fut simplement une guerre des miséreux contre les nantis, des pauvres contre les riches ${ }^{25}$.

$\mathrm{Au}$ surplus, il conviendrait d'établir à cet égard une distinction rigoureuse entre le périmètre où s'exerce depuis 1791 l'influence politique des «Arpajonnais » et les autres zones touchées par les troubles de 1792 :

Il ne faudrait pourtant pas exagérer les aspects politiques de la jacquerie : si, dans le canton d'Aurillac, le mouvement de protestation paysanne avait été largement organisé et politisé, depuis l'année précédente, par l'intermédiaire du club d'Arpajon et de la fédération de gardes nationales de Milhaud, hors de ce canton, l'insurrection prit une allure plus spontanée, exprimant de manière plus directe, moins complexe, les griefs particuliers locaux. En Châtaigneraie, c'est le ressentiment éveillé par les souvenirs du régime féodal qui se manifesta ; dans le district de Saint-Flour, le conflit religieux eut tendance à l'emporter ${ }^{26}$.

Deux postulats implicites affleurent dans ce propos en manière de bilan. Le premier tient qu'il est possible d'isoler le mobile d'une action à partir des formes particulières qu'elle revêt. Le second suppose l'existence d'une démarcation nette, sinon étanche, entre ce qui relève de la « grande politique », de portée nationale, et ce qui ressortit à une «politique du peuple », d'intérêt strictement local.

\footnotetext{
22 Anatoli ADO, Paysans en Révolution..., op. cit., p. 293.

23 Ibid., p. 293.

${ }^{24}$ Ibid., p. 304.

${ }^{25}$ Jonathan R. DALBY, Les Paysans cantaliens..., op. cit., p. 74 et 76.

26 Ibid., p. 81.
} 
Il serait certes vain de prêter une charge idéologique à l'ensemble des faits et gestes constitutifs de l'insurrection cantalienne de 1792. Mais il semble non moins réducteur de dissocier trop fortement, in abstracto, ce qui était inextricablement lié dans la conscience des contemporains.

\section{Du seigneur opulent à l'aristocrate comploteur : des réactions punitives contre une figure familière de l'ennemi.}

\section{L’inextricable écheveau des motivations.}

Transportons-nous hors du canton et même du district d'Aurillac, et considérons d'abord les faits séditieux dénoncés par le directeur du jury du tribunal de district de SaintFlour dans un acte d'accusation du 24 juillet 1792, concernant plusieurs habitants de Malbo :

(...) le dix huit du mois de mars dernier, une troupe dhommes armés au nombre d'environ deux cent arriva sur le soir au lieu de Lescure parroisse de St martin et sintroduisirent a force ouverte chés le Sr de Lastic, quils forcerent a leur donner a boire et a manger a discretion, que non contents de ce quon leur servoit, ils se saisirent de tous les comestibles quils trouverent dans la maison, semparerent de la cave et firent de tout une de lapidation, que bientot après ils exigerent $d u \mathrm{Sr}$ de Lastic quil leur remit trente fuzils quils disoient etre dans sa maison, que d'après limpossibité (sic) du Sr de Lastic de delivrer les trente fuzils quil $n$ avoit pas ils le menacerent de luy couper la tete, quils forcerent et briserent toutes les portes des appartements et armoires du Sr de Lastic sous pretexte de rentte, sil ny avoit pas de fuzils de cachés, mais que leur vray but etoit de voler et pilier $(\ldots)^{27}$.

Si, à l'instar de B. de Miramon-Fargues, les autorités judiciaires ne veulent voir dans cette visite du château de Lescure (Saint-Martin-sous-Vigouroux) qu'une simple opération de pillage, les actes et mobiles rapportés suggèrent une pluralité de préoccupations, sans qu'il soit aisé d'établir précisément les correspondances entre les premiers et les secondes : la demande de denrées alimentaires répond-elle à des besoins frumentaires et/ou à la volonté de précipiter et de mettre en scène, au domicile même du ci-devant seigneur, l'avènement d'un nouvel ordre des $\operatorname{choses}^{28}$ ? La recherche de fusils cachés dénote-t-elle la crainte d'un « complot aristocratique » et/ou le souci de prendre une revanche sur (et chez) celui qui faisait

\footnotetext{
27 A.D.C., L 1433.

${ }^{28}$ John MARKOFF note à ce sujet que «Si un groupe de ruraux envahit le château du seigneur et engage ce dernier, d'un ton menaçant, à les nourrir, s'agit-il avant tout d'une invocation de la règle d'hospitalité, parodiée de façon moqueuse afin de prouver que ceux qui dépendaient jadis du seigneur sont désormais ceux qui font la loi ? S'agit-il d'une action «antiseigneuriale» ou «de subsistance»?» («Violence, Emancipation, and Democracy: The Countryside and the French Revolution », The American Historical Review, vol. 100, n² 2, 1995, p. 369, n. 34).
} 
jadis perquisitionner les maisons paysannes pour y débusquer armes prohibées et criminels dissimulés ${ }^{29}$ ?

Ailleurs, le répertoire d'actions n'est pas toujours aussi diversifié. Mais, même quand on se contente de satisfaire «le boire et le manger $»^{30}$, les motivations antiseigneuriales et patriotiques sont-elles vraiment absentes ?

Toujours est-il que l'imbrication des registres politique, social et architectural, est parfaitement perçue par le sieur Fau, administrateur du district d'Aurillac, quand il se livre, pour en désamorcer les effets dévastateurs, à une redéfinition du château. Alors que la fermentation règne parmi les hommes attroupés devant le château de Longuevergne (Leynhac), Fau :

(...) leur a représenté quele pillage et les dévastations étaient prohibées[,] que ce serait d'ailleurs une perte pour tous les citoyens si l'on détruisait les maisons qui servent d'asile particulierement aux cultivateurs et que les chateaux n'étaient à proprement parler que des maisons destinées à cet usage $e^{31}$.

Tout en combattant l'exclusion des châteaux de la communauté rurale, le discours attribué à Fau témoigne de l'abîme qui sépare désormais les nobles-aristocrates-châtelains et les populations environnantes : tout se passe comme si la préservation des propriétés des premiers dépendait nécessairement de leur dépossession symbolique au profit des secondes.

Une telle «politisation» du château consacre l'émergence dans les campagnes cantaliennes d'une nouvelle culture politique, qui trouve aussi à s'exprimer dans divers rites et emblèmes patriotiques, destinés à remplacer la macule seigneuriale et nobiliaire dans le paysage quotidien.

\section{A l'encontre d'une sémiotique châtelaine séculaire, une « politisation des images et des couleurs $»^{32}$.}

Ainsi, s'étant rendu sur la place de Junhac le 20 mars, un laboureur «trouva un attroupement de gens de lad[ite] parroisse dont plusieurs luy dirent, de sen revenir ches luy, et de les rejoindre promptement a Goudergnes avec son fuzil, une coquarde et un sac $»^{33}$. Avant de se mettre en marche, le commandant de la garde nationale de Junhac leur « fait preter le serment d'etre fidelles a la nation[,] a la loy, et au roy $»^{34}$.

\footnotetext{
${ }^{29}$ John MARKOFF, «Violence, Emancipation... », art. cit., p. 371.

${ }^{30}$ Expression employée dans le procès-verbal de la municipalité de Vitrac du 22 mars 1792 (A.N. F7 3662).

31 A.N. F7 3662, "procès verbal de troubles » de la municipalité de Leynhac, 21-22 mars 1792.

32 Yves-Marie BERCE, Croquants et nu-pieds..., op. cit., p. 169.

33 A.D.C., L 1587, Justice de paix du canton de Montsalvy, Témoignage n 32, 23 mai 1792.

34 A.D.C., L 1587, Just. de p. de Montsalvy, Tém. nº 18, 21 mai 1792.
} 
Les «objectivations symboliques» par lesquelles se manifestent des «plaintes théâtralement matérialisées ${ }^{35}$ concernent aussi les emblèmes honnis d'un monde qui n'en finit pas de mourir. A Parlan, le 22 mars, la troupe massée auprès du château paraît apaisée :

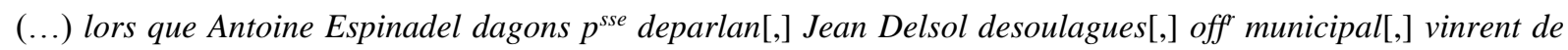
Roumegoux avec un charpantie quil croit etre dela $p^{\text {sse }}$ de Quezac et dirent publiquement en levant la main qu'on navoit pas asses fait au chateau deparlan et que si on se bornoit aladescente des giroettes, quatre cents hommes alloient venir sur la parroisse pour tout leraser ; que lepeuple leva aussy la main deson cotte et chanta ensuitte de chansons patriotiques. que le charpentier qui etoit venu avec led[it] Spinadel et Delsol portoit a sa main un baton entouré d un ruban rouge et couronnè d un debris de giroette ${ }^{36}$.

A l'occasion, les rituels patriotiques entourant les attaques de châteaux recourent ainsi à des insignes voués à l'exécration populaire, ici un morceau de girouette et un ruban distinctif de l'ordre de Saint-Louis.

La mobilisation d'une symbolique de caractère national, qu'elle sanctionne l'adhésion ou l'opposition à la Révolution, ne doit néanmoins pas faire illusion : les révoltés s'en prennent d'abord à une figure familière de l'ennemi, et non point à un adversaire-type abstrait.

\section{Impulsions extérieures et mobilisations locales pour des luttes sélectives.}

Même si le directoire du district d'Aurillac a pu parler d'une «insurrection générale $»^{37}$, les opérations menées s'avèrent éminemment sélectives. Sans surprise, le château de Vixouze (Polminhac) est épargné par l'insurrection, tandis que celui de Niocel (Marmanhac) «a ete brulé le 17 mars 1792 par une troupe de brigands organisés par les factieux qui regnoient dans ces temps horribles $»^{38}$. Pour l'ensemble du district d'Aurillac, «plus de 30 châteaux ont été visités, dont une demi-douzaine incendiés ${ }^{39}$, chiffres qui demeurent somme toute modestes quand on les rapporte aux 142 édifices mentionnés sur la carte de $\mathrm{Cassini}^{40}$. Au reste, à l'échelle départementale, les cartes insérées en fin de

\footnotetext{
35 Yves-Marie BERCE, Croquants et nu-pieds..., op. cit., p. 169.

36 A.D.C., L 1582, Just. de p. de Maurs, Tém. nº 20 (officier municipal de Parlan), 26 avril 1792.

${ }^{37}$ Dans deux documents conservés aux Archives nationales, cités dans Hubert DeLPONT, La Victoire des croquants..., op. cit., p. 239, n. 1.

38 B.C.I.U. de Clermont-Ferrand, Fonds Patrimoine, MS 510, [Deval DE SAUnADE], Geographie de touts les chateaux, des cy devant fiefs, maisons de campagne, et autres lieux remarquables, t. 2, entrée « Niocel », p. 74.

${ }^{39}$ Hubert DeLPONT, La Victoire des croquants..., op. cit., p. 242.

40 Ce total comprend 101 «châteaux », 31 "gentilhommières, fiefs, maisons de plaisance, ou de campagne », 7 " châteaux ruinés » et 3 châteaux identifiés par la seule toponymie, car associés au symbole du hameau. La Geographie... de Deval de Saunade, sorte de dictionnaire des châteaux auvergnats rédigé au tournant des XVIII ${ }^{\mathrm{e}}$ et XIX $^{\mathrm{e}}$ siècles, signale pour sa part, sur l'étendue du district d'Aurillac, 153 demeures châtelaines, dont 117 « châteaux », 24 « maisons de campagne, jadis fief » et 12 « châteaux ruinés ».
} 
contribution $^{41}$ font apparaître des «trouées » significatives dans la distribution des communes touchées par les attaques de châteaux, et révèlent que bien souvent, dans une commune donnée, tous les châteaux indiqués sur la carte de Cassini ne sont pas visités en mars 1792. Il semble ainsi que l'exaspération des ruraux s'est concentrée sur des lieux et des personnes bien connus, contre lesquels s'élevait un faisceau de griefs enracinés dans un «passé qui ne passe pas », et qui, en s'étoffant au fil des mois en liaison avec l'évolution de la conjoncture nationale, ont fini par former un substrat mental cohérent, rendant pensable, donc possible, l'action subversive.

Le recrutement majoritairement local des émeutiers conforte cette impression. Bien des procédures judiciaires corrigent en effet l'image laissée par les procès-verbaux contemporains des événements, dans lesquels les municipalités, soucieuses de se dédouaner vis-à-vis des autorités supérieures du district ou département, ont tendance à « rejeter la principale responsabilité des événements sur ceux qui sont venus de l'extérieur de la commune $»^{42}$.

Il ne faudrait pourtant pas sous-estimer l'influence des «étrangers » sur le cours des événements. Lorsqu'ils ne prennent pas une part directe aux luttes, ils nourrissent certainement les imaginaires, attisant les espérances ou les peurs, et fournissent opportunément un exemple à suivre, paré des vertus légitimatrices du précédent. De fait, l'annonce d'une incursion extérieure imminente, que la menace soit réelle ou fictive, précipite souvent le dénouement des tensions ${ }^{43}$, en ce qu'elle catalyse l'épanchement des divisions internes de la communauté rurale.

\section{Entre notabilité et liberté de ton : un ennemi commun, mais deux visions concurrentes de la justice sociale.}

\section{Des communes divisées sur la peine à infliger.}

\footnotetext{
${ }^{41}$ Aux événements recensés par Hubert Delpont, qui fournit l'inventaire le plus complet, ont été ajoutés les incidents survenus aux châteaux de Niocel, de Cours et de Polvrières. Au total, ce ne sont ainsi pas moins de 44 châteaux, répartis dans 32 communes, qui ont été attaqués lors des troubles cantaliens de mars 1792.

${ }^{42}$ Jean VEzole, «A propos des troubles ruraux dans le Cantal en 1792 : les incidents de Glénat (21-22 mars) », Revue de la Haute-Auvergne, 1989, p. 281.

${ }^{43}$ Ce que suggérait le témoignage cité plus haut, évoquant la mise en échec des mesures d'apaisement prises par la municipalité de Parlan après l'irruption de trois personnes, dont un officier municipal, qui brandissent la menace d'une invasion de quatre cents hommes.
} 
Le soulèvement cantalien présente, certes, d'indéniables aspects festifs, l'ivresse collective, au propre comme au figuré, constituant ici ou là «l'aboutissement attendu, le couronnement délirant d'un jour extraordinaire $»^{44}$. Mais les sources judiciaires jettent aussi une lumière vive sur les divergences idéologiques qui traversent le monde rural. L'hostilité partagée envers la figure du noble aristocrate ne débouche pas, en certains endroits du moins, sur des rivalités mettant aux prises deux forces antagonistes, mais sur des «triangulaires » mettant en jeu deux conceptions de la justice sociale ${ }^{45}$.

Dans le feu de l'action, les dissensions intestines peuvent revêtir une grande intensité dramatique. Tandis que les invectives pleuvent sur les plus timorés, les autorités locales sont parfois contestées à force ouverte.

Il semble toutefois que les rapports de force décidant de l'action collective tiennent moins de la violence physique que de la joute oratoire, ainsi que le suggère le débat auquel a assisté ce laboureur du lieu de Junhac, chez lequel une partie de l'attroupement formé la veille a passé la nuit du 21 mars :

(...) a l'aurore le declarant ayant entendu un murmure dans sa maison entre ceux qui y avaint passé la nuit, et s'etant informé du sujet il entendit confusement que les uns pensaint quil falait mettre lefeu au chateau dud Junhac et les autres que non et que tous s'accordaint pour dire quil falait partager les effets, que led fau maCouvreur soutint avec oppiniatreté quil falait bruler les papiers, que le $S^{r}$ Goudergnes fils cadet [qui commande alors la garde nationale] dit que sil etait question de mettre le feu il se demetait desa place, et led delom ayné [fermier au domaine du château de Junhac] quil offrirait plutot mille ecus que de permettre le feu, que certains parurent accepter cette offre et les autres la rejetterent $t^{46}$.

Les déchirements des communautés, qui culminent lors du pillage ou de l'incendie du château, paraissent ainsi procéder, dans une large mesure, d'efforts de persuasion déployés concurremment par différents «meneurs». De telles prises de parole, que l'on devine animées, sont assurément tributaires de ce que Maurice Agulhon nomme «le poids empirique des réalités contingentes », constituées ici par les sollicitations extérieures, mais celles-ci n'expliquent pas tout. Car derrière les désaccords sur la sanction à infliger, se profilent deux visions profondément différentes d'un ordre politique et social débarrassé de la domination féodo-nobiliaire.

\footnotetext{
${ }^{44}$ Yves-Marie BERCE, Croquants et nu-pieds..., op. cit., p. 102.

45 Après avoir étudié les multiples conflits qui opposent, dans la Corrèze des années 1790-1792, les paysans aux seigneurs devenus aristocrates, Jean BOUTIER consacre un développement aux tensions « au sein des communautés ", examinées "à travers les rares échos que nous renvoient les délibérations départementales 》 (Campagnes en émoi. Révoltes et Révolution en Bas-Limousin. 1789-1800, Treignac, Éditions «Les Monédières », 1987, p. 168). Les deux séries de faits apparaissent alors comme étrangères l'une à l'autre, ne coïncidant ni dans la chronologie, ni dans le rapport au politique: «A la différence des grandes vagues de révoltes, dont la répercussion est souvent nationale, cette agitation diffuse des campagnes reste le plus souvent sans lendemain » (ibid., p. 171). Nos sources judiciaires incitent à lier plus fortement les deux dynamiques contestataires.

46 A.D.C., L 1587, Just. de p. de Montsalvy, Tém. n 9, 19 mai 1792.
} 


\section{Une conception « verticale » des rapports sociaux dans un monde roturier.}

Cette première ligne politique est incarnée d'abord par les « cadres institutionnels ${ }^{47}$, officiers municipaux et surtout gardes nationaux, qui sont généralement à l'origine de la mobilisation locale. Leur puissance économique et sociale, qui s'appuie certainement sur des liens familiaux et clientélaires similaires à ceux tissés par les fermiers artésiens de Jean-Pierre Jessenne, a trouvé une reconnaissance, la Révolution venue, sur le plan politique: les municipalités ont hérité d'une partie substantielle des pouvoirs de police seigneuriaux et les gardes nationales sont devenues les principales forces de l'ordre dans les campagnes. A ce titre, ces «notables » ont tout à craindre d'une Contre-Révolution nobiliaire et se joignent sans états d'âme à l'animosité ambiante contre le monde châtelain. Mais, dans une société désormais roturière, ils entendent bien fonder leur prééminence sur la hiérarchie des fortunes et des pouvoirs, comme l'atteste leur conduite devant la radicalisation du soulèvement.

«Le boire et le manger» dans les murs du château les comblent, en ce que cette appropriation des lieux consacre, sur un mode spectaculaire et festif, le transfert du «pouvoir au village » survenu depuis $1789^{48}$. Ils s'accommodent volontiers, les circonstances aidant, de la descente des girouettes, voire du nivellement des tours, ramenées à la hauteur du toit du corps principal : il est vrai que «Ses tourelles abattues et ses armoiries détruites, le château n'est plus qu'une maison $»^{49}$ dépouillée des attributs d'une suprématie séculaire heureusement révolue. Ils s'opposent en revanche avec véhémence au pillage et à l'incendie du château, car ce n'est plus alors la dimension politique de la seigneurie qui se trouve visée, mais bien l'opulence d'une demeure et, à travers celle-ci, le droit de propriété et les inégalités de revenu. A tout prendre, cependant, ils préfèrent encore voir partir en flammes le château plutôt que leur habitation. La dernière chose que leur pragmatisme à toute épreuve refuse de sacrifier, c'est le chartrier. Le « terrier constituant non seulement un titre de féodalité pour le seigneur,

\footnotetext{
47 Pour reprendre la typologie des «moteurs » du mouvement paysan picard proposée par Florence GAUTHIER et Guy-Robert IKNI, dans «Le mouvement paysan en Picardie: meneurs, pratiques, maturation et signification historique d'un programme (1775-1794)», dans Jean NiCOLAS (dir.), Mouvements populaires et conscience sociale. XVI ${ }^{e}$ XIX ${ }^{e}$ siècles [Actes du colloque de Paris, 24-26 mai 1984], [Paris], Maloine Éditeur, 1985, p. 437-438.

${ }^{48}$ De ce point de vue, les intrusions dans les châteaux lors de l'insurrection de 1792 peuvent être rapprochées d'autres formes de «violation de l'espace nobiliaire » sous la Révolution, telles que les visites domiciliaires à des fins d'inventaire, de perquisition ou de réquisition, prévues par la législation révolutionnaire (Philippe GRANDCOING, Les Demeures de la distinction. Châteaux et châtelains au XIX siècle en Haute-Vienne, Limoges, PULIM, 1999, p. 109-111).

${ }^{49}$ John MARKOFF, « Violence, Emancipation... », art. cit., p. 372.
} 
mais aussi un titre de propriété pour les tenanciers $»^{50}$, ils devaient être particulièrement sensibles à ce dernier aspect, qui les concernait et préoccupait finalement davantage que le château lui-même. Delolm aîné n'en fait pas mystère à ce laboureur qui :

(...) vit un attroupement audedans et au dehors dud chateau de Junhac et qu'ayant parlé avec led delom ayné pour scavoir de luy, ce qu'on voulait faire et si M. figeagol avait ecrit qu'on brulat led chateau, aquoy Delon repondit, passe pour le chateau, mais il faudrait au moins conserver les papiers, et quil y en avait trois qui voulaint les bruler ${ }^{51}$.

\section{Une conception «horizontale » du lien social en terre d'égalité.}

Ces trois «meneurs organiques $»^{52}$, qui veulent à toute fin brûler les papiers et incendier le château, se font les défenseurs d'une ligne politique spécifique, proche de l'«économie morale de la foule» analysée par Edward P. Thompson et du programme égalitaire des paysans picards mis au jour par F. Gauthier et G.-R. Ikni : dans le Cantal aussi, cet «égalitarisme fut avant tout l'expression la plus radicale de l'antiféodalisme, mais, en outre, ce courant exprime une conception originale de la révolution, du progrès économique et du droit. Les couches inférieures refusent de changer de maîtres. L'aristocratie féodale étant tombée [ou en passe de l'être], elles refusent de se soumettre à celle des riches ${ }^{53}$. Leur fort engagement dans la discussion et l'action, à un moment où la distinction entre citoyens actifs et citoyens passifs continue de régir l'accès au vote et à la garde nationale, annonce assez leur vision de l'ordre politique : c'est vers la démocratie directe, appuyée sur le mandat impératif, que doit aller leur prédilection.

Lors des attaques de châteaux, deux formes comportementales manifestent la quête d'un nivellement économique et social. L'une, empreinte de nihilisme, se caractérise par une soif de destruction, qui amène les révoltés de Malbo, par exemple, à refuser « de prendre les clefs des apartements [du château de Lescure,] preferant de les enfoncer ${ }^{54}$. L'autre manifeste un souci de redistribution, bien distinct de la simple saisie d'effets entachée d'opportunisme. Emblématique de cette attitude est la ferme volonté de partage des meubles du château de Naucaze (Saint-Julien-de-Toursac) affichée par un «travailleur » de l'endroit :

\footnotetext{
50 Albert SOBOUL, «Le brûlement des titres féodaux (1789-1793) » [article paru à l'origine dans Annales historiques de la Révolution française, 1964], dans A. SobOul, Problèmes paysans de la Révolution, 1789-1848, Paris, La Découverte, 2001, p. 144-145.

51 A.D.C., L 1587, Just. de p. de Montsalvy, Tém. nº 31, 23 mai 1792.

${ }^{52}$ Florence GAUTHIER et Guy-Robert IKNI, « Le mouvement paysan... », art. cit., p. 437-438.

${ }^{53}$ Ibid., p. 443.

${ }^{54}$ A.D.C., L 1433, Tribunal de district de Saint-Flour, Tém. n 1, 6 juillet 1792.
} 
Led Blanquies sagitant au tour du peuple pretandit quon navoit pas besoin deboire, quil falloit faire a linstant lepartage detous les meubles. que ce propos exita une fermentation generalle que la municipalitte parvint cepandant a arreter $^{55}$.

Ces deux modes d'action contradictoires visant à « faire passer sur les maisons de la République le niveau de l'égalité ${ }^{56}$ ne paraissent pas réductibles à une «guerre sociale, pauvres contre riches ${ }^{57}$. Si l'on considère l'affaire qui a impliqué le plus grand nombre de prévenus, à savoir le procès des 40 hommes accusés d'avoir participé aux attaques de quatre châteaux situés sur les communes de Junhac et de Sénezergues, il apparaît que 31 d'entre eux sont déclarés non coupables par les jurés du tribunal de district d'Aurillac, qui condamnent en revanche les 9 autres. Au nombre des premiers, se rangent notamment les «notables» instigateurs de l'attroupement de Junhac (Delolm aîné et le « sieur » Goudergnes), tandis que le second groupe comprend les trois «meneurs organiques » promoteurs de l'incendie des papiers et du château. Mais, pour autant que les pièces de la procédure ${ }^{58}$ permettent d'en juger, les deux ensembles d'émeutiers distingués par la justice se recrutent dans des milieux socioprofessionnels variés et pas essentiellement différents. Outre le couvreur Fau, on compte ainsi parmi les 9 «enragés » ${ }^{59}$ le « sieur » Vaissière, cabaretier de Labesserette se déplaçant à cheval, et le propre frère de Delolm aîné; dans l'autre groupe, on trouve, aux côtés de plusieurs laboureurs, de deux meuniers, d'un tisserand et d'un sabotier, etc., deux bouviers, dont l'un est employé par l'un des laboureurs précités. Il semble donc que les autorités judiciaires, refusant une condamnation en bloc qui risquerait de relancer l'agitation, ont concentré leurs coups sur les insurgés les plus dangereux plutôt que sur des catégories sociales données. Les plus dangereux, car ils incarnent la ligne politique la plus radicale au sein de ruraux ayant pourtant un adversaire commun : le châtelain, noble et aristocrate.

Ainsi, dans les campagnes cantaliennes de mars 1792, la figure de l'ennemi aristocrate divise autant qu'elle rassemble. Ce faisant, elle contribue doublement à la politisation du monde rural : d'un côté, la mobilisation communautaire contre les châteaux du voisinage, en ce qu'elle instaure et met en scène la relation unissant griefs particuliers et conjoncture

\footnotetext{
55 A.D.C., L 1590, Just. de p. de Maurs, Tém. nº 5 (concierge du château de Naucaze), 27 avril 1792.

56 Objectif que rejette explicitement la Convention dans son décret du 13 pluviôse an II (1 ${ }^{\text {er }}$ février 1794), qui normalise la démolition des « châteaux-forts et forteresses de l'intérieur » envisagée depuis l'été 1793 (cité dans JeanPaul FANGET, «La démolition révolutionnaire des châteaux féodaux dans le département du Puy-de-Dôme. 1789-an II », Revue d'Auvergne, 1979, p. 376).

${ }^{57}$ Pour reprendre la formule employée par Daniel MARTIN (La Révolution en Auvergne, Bourbonnais et Velay, ClermontFerrand, Bouhdiba, 1993, p. 198).

58 A.D.C., L 1587. Les châteaux concernés sont ceux de Cours, Polvrières et Sénezergues (commune de Sénezergues) et celui de Junhac.

${ }^{59}$ Le terme est utilisé dans l'acte d'accusation (du 12 juillet 1792) présenté par le directeur du jury du tribunal de district d'Aurillac (A.D.C., L 1587).
} 
générale, tend à approfondir « l'intégration nationale »; de l'autre, les tensions entre roturiers, dans la mesure où elles s'enracinent dans deux visions politiques concurrentes et qu'elles associent dans cette confrontation de larges pans de la société locale (citoyens actifs et passifs, hommes et femmes, jeunes et vieux), participent du processus de «transition démocratique $»^{60}$.

Ces mouvements de politisation ne sont nullement l'apanage du canton d'Aurillac. On peut même penser, en manière de paradoxe, qu'ils atteignent leur intensité maximale là où les troupes de la fédération arpajonnaise n'interviennent pas directement, car s'ouvrent alors des possibilités de «triangulaires» propices au débat démocratique, quelques mois avant la proclamation de la République.

Sébastien Pivoteau

\footnotetext{
${ }^{60}$ Ces deux dynamiques, l'intégration nationale et la transition démocratique, constituent les deux axes privilégiés par l'historiographie de «La politisation des paysans au XIX ${ }^{\mathrm{e}}$ siècle » analysée par Gilles PECOUT dans Histoire et Sociétés rurales, $\mathrm{n}^{\circ} 2$, 1994, p. 92-96.
} 
Les attaques de châteaux lors des troubles cantaliens de mars 1792 : des opérations sélectives.

1) Communes où des châteaux ont été attaqués en mars 1792.

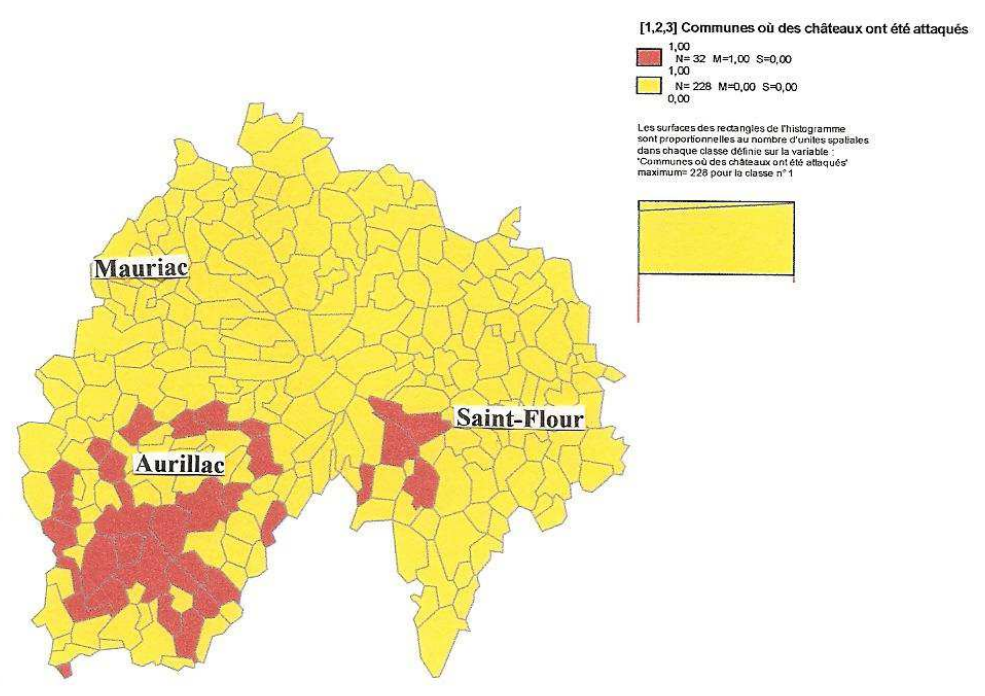

Fait avec Phicarto * 26108/2008 12:32:18 * http://phiggeo.club.ff

2) Nombre de châteaux attaqués en mars 1792, rapporté au nombre de châteaux mentionnés sur la carte de Cassini.

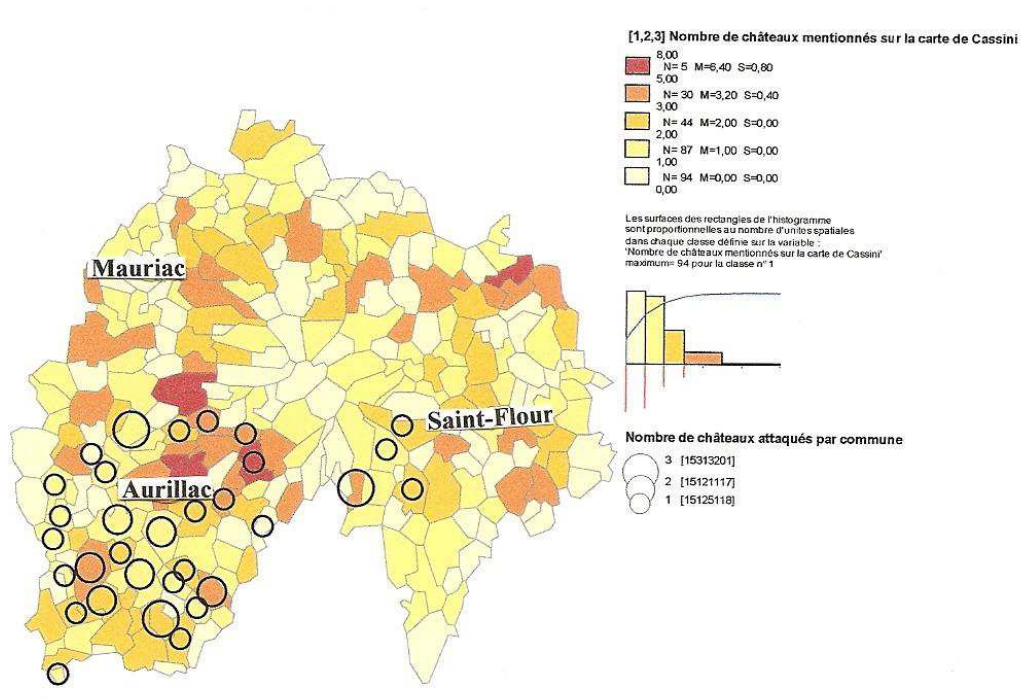

